\title{
ON DECOMPOSITIONS OF 3-SPACE BY LINKAGES
}

\author{
R. P. GOBLIRSCH
}

1. Introduction. A linkage is a finite set of disjoint simple closed curves in 3-space. The space of a linkage is the decomposition space associated with the decomposition of 3-space whose only nondegenerate elements are the simple closed curves of the linkage. R. H. Bing and M. L. Curtis have shown that there is a linkage of nine circles whose space is not imbeddable in 4-space. They conjecture that the space of a linkage consisting of a set of three circles each pair of which is linked is not imbeddable in 4-space. (See Abstract 551-9 Notices Amer. Math. Soc. volume 5 (1958).) We show that this conjecture is incorrect and that the spaces of several linkages of three simple closed curves can be imbedded in 4-space.

2. A description of the linkages. Let $R$ be the field of real numbers and let $C$ be the field of complex numbers. For each positive integer $N$, let $N$-space, $R^{N}$, be the space of all functions $x$ from the set of the first $N$ positive integers to $R$ with $|x|=\left(\sum_{k=1}^{N}\left|x_{k}\right|^{2}\right)^{1 / 2}$, where $x_{k}$ is the image of $k$ by $x$. If $R$ is replaced by $C$, we obtain complex $N$-space, $C^{N}$. We identify $R^{2 N}$ with $C^{N}$ by the correspondence $z_{k}=x_{2 k-1}+i x_{2 k}$. We consider that $R^{N}$ is imbedded in $R^{N+1}$ as the hyperplane $\left\{x \in R^{N+1}: x_{N+1}=0\right\}$. Let $S^{N}=\left\{x \in R^{N+1}:|x|=1\right\}, N=0$, $1, \cdots$. Finally, let $T$ be the torus $\left\{z \in C^{2}:\left|z_{1}\right|=\left|z_{2}\right|=1\right\}$.

If $z \in S^{3}$, there is exactly one number $\alpha(z), 0 \leqq \alpha(z) \leqq \pi / 2$, so that $\cos \alpha(z)=\left|z_{1}\right|$ and $\sin \alpha(z)=\left|z_{2}\right|$. Let $J_{1}=\left\{z \in S^{3}: \alpha(z)=0\right\}$ and $J_{2}=\left\{z \in S^{3}: z_{1}=1 / 2\right\}$. Then $J_{1}$ and $J_{2}$ are simple closed curves in $S^{3}$. For each number $a, 0<a<\pi / 2, T_{a}=\left\{z \in S^{3}: \alpha(z)=a\right\}$ is a torus homeomorphic with $T$ under the map $z \rightarrow\left(z_{1} /\left|z_{1}\right|, z_{2} /\left|z_{2}\right|\right)$. Let $J_{3}$ be any simple closed curve on $T_{\pi / 4}$ which does not separate $T_{\pi / 4}$.

The linkages in $R^{3}$ which we shall use are obtained from $J_{1}, J_{2}$ and $J_{3}$ by a stereographic projection of $S^{3}$ on $R^{3}$. Let $w=(0,0,0,1)$ and let $\pi: S^{3}-w \rightarrow R^{3}$ be defined by $\pi(x)=\left(2 /\left(1-x_{4}\right)\right)\left(x_{1}, x_{2}, x_{3}\right)$. Then $\pi J_{1}$ is a circle in the $\left(x_{1}, x_{2}\right)$-plane, and $\pi J_{2}$ is a circle in the $\left(x_{1}, x_{3}\right)$ plane which links $\pi J_{1}, \pi J_{3}$ is a simple closed curve which may link, but not necessarily simply, both $\pi J_{1}$ and $\pi J_{2}$. If $J_{3}=\left\{z \in S^{3}: z_{1}=z_{2}\right\}$, then $\pi J_{3}$ is a circle in the plane $x_{1}=x_{3}$ which links both $\pi J_{1}$ and $\pi J_{2}$, and we have the case in the conjecture of Bing and Curtis.

Presented to the Society, April 24, 1959 under the title On imbedding decompositions of 3-space in 4-space; received by the editors December 8, 1958. 
3. In which we imbed the spaces of these linkages in 4-space. Let $X$ be the space of the linkage consisting of $\pi J_{1}, \pi J_{2}$ and $\pi J_{3}$, and let $X^{\prime}$ be the decomposition space of $S^{3}$ whose nondegenerate elements are $J_{1}, J_{2}$ and $J_{3}$. Suppose that $X^{\prime}$ is imbedded in $R^{4}$. Then we imbed $X^{\prime}$ in $S^{4}$ by compactifying $R^{4}$ with a point at infinity. Now remove the image of the point $w \in S^{3}$ from $S^{4}$, and so from $X^{\prime}$. We find that $X$ is imbedded in $R^{4}$.

Let $J_{2}^{\prime}=\left\{z \in S^{3}: \alpha(z)=\pi / 2\right\}$. Then $J_{2}^{\prime}$ is a simple closed curve in $S^{3}$. Moreover, there is a homeomorphism of $S^{3}$ onto itself which maps $J_{2}^{\prime}$ onto $J_{2}$ and which leaves $J_{1}$ and $T_{\pi / 4}$ fixed. Hence $X^{\prime}$ is homeomorphic to the decomposition space of $S^{3}$ whose only nondegenerate elements are $J_{1}, J_{2}^{\prime}$ and $J_{3}$.

Let $J_{3}^{\prime}$ be the image of $J_{3}$ under the mapping $T_{\pi / 4} \rightarrow T$ previously described. Since $J_{3}^{\prime}$ does not separate $T$, there is a homeomorphism $f: T \rightarrow R^{3}$ such that $f$ maps $J_{3}^{\prime}$ onto $\left\{x \in R^{3}:|x|=1, x_{3}=0\right\}$ and $f$ maps $T$ onto the torus obtained by revolving the circle $\left\{x \in R^{3}: \mid x\right.$ $\left.-(2,0,0) \mid=1, x_{2}=0\right\}$ around the $x_{3}$-axis.

The join, $S^{0} * T$, of $S^{0}$ and $T$ is the decomposition space of $S^{0} \times T$ $\times[0,1]$ whose only nondegenerate elements are $\left\{\left(S^{0}, z, 0\right): z \in T\right\}$ and $\left\{(p, T, 1): p \in S^{0}\right\}$. If $(p, x, t) \in S^{0} \times T \times[0,1]$, let $\langle p, x, t\rangle$ be the element of $S^{0} * T$ which contains it. Define $\Phi: S^{3} \rightarrow S^{0} * T$ by

$$
\begin{aligned}
& \Phi(z)=\left\langle 1,\left(z_{1} /\left|z_{1}\right|, z_{2} /\left|z_{2}\right|\right), 1-\frac{4}{\pi} \alpha(z)\right\rangle \text { if } 0<\alpha(z) \leqq \frac{\pi}{4}, \\
& \Phi(z)=\left\langle-1,\left(z_{1} /\left|z_{1}\right|, z_{2} /\left|z_{2}\right|\right), \frac{4}{\pi} \alpha(z)-1\right\rangle \text { if } \frac{\pi}{4} \leqq \alpha(z)<\frac{\pi}{2}, \\
& \Phi(z)=\langle 1, z, 1\rangle \text { if } \alpha=0,
\end{aligned}
$$

and

$$
\Phi(z)=\langle-1, z, 1\rangle \text { if } \alpha=\pi / 2 .
$$

Then $\Phi$ is continuous and the decomposition of $S^{3}$ associated with $\Phi$ has for its nondegenerate elements $J_{1}$ and $J_{2}^{\prime}$. The decomposition space is homeomorphic with $S^{0} * T$.

We now imbed $S^{0} * T$ in $R^{4}$. Let

$$
\begin{aligned}
F(1, T, 1) & =(0,0,0,1), \\
F(-1, T, 1) & =(0,0,0,-1),
\end{aligned}
$$

and

$$
F(p, x, t)=t F(p, T, 1)+(1-t) f(x) \text { if } 0 \leqq t<1,
$$


where $f: T \rightarrow R^{3}$ is the homeomorphism defined earlier. This $F$ is an imbedding of $S^{0} * T$ in $R^{4}$.

It is clear that there is a mapping $G: R^{4} \rightarrow R^{4}$ such that the associated decomposition space of $R^{4}$ has for its only nondegenerate element the disc $\left\{x \in R^{4}:|x| \leqq 1, x_{3}=x_{4}=0\right\}$. The map $G F \Phi: S^{3} \rightarrow R^{4}$ determines an imbedding of $X^{\prime}$ in 4 -space. Therefore we have supported the claims of the introduction.

Let $K$ be a proper closed subset of $S^{1}$, and for each $t \in K$ let $J_{3}(t)$ $=\left\{z \in S^{3}: z_{1}=t z_{2}\right\}$. Then $\left\{\pi J_{3}(t): t \in K\right\}$ is a family of pairwise linking circles in $R^{3}$. By modifying $\Phi, F$ and $G$ slightly one can imbed the space of this linkage in $R^{4}$.

UNIVERSITY OF VIRGINIA

\section{A NOTE ON A THEOREM OF LIAO}

\section{P. E. CONNER}

The purpose of this note is the presentation of a theorem announced by S. D. Liao [3]. After the submission of this note a different proof, by Liao, appeared [4]. We shall state

Theorem 1. Let $\left(S^{1}, S^{n}\right)$ denote the circle group operating as a topological transformation group on the n-sphere. If $M^{r}$ and $N^{n-r-1}$ are two closed, disjoint, invariant submanifolds of $\left(S^{1}, S^{n}\right)$ which have a nonzero linking number, then if $M^{r}$ contains no stationary points, it follows that $N^{n-r-1}$ contains all the stationary points.

Actually, Liao stated the theorem for the action of a cyclic group of prime order, $Z_{p}$, on the sphere. In that case, it is necessary to assume that the invariant submanifolds are linked mod $p$, otherwise the principle of proof is the same. We shall denote the circle group acting on a locally compact, connected, separable metric space $X$ by $\left(S^{1}, X\right)$. We shall denote by $F \subset X$ the set of points in $X$ fixed under the entire group. Throughout this note we shall use AlexanderWallace-Spanier cohomology groups with rational coefficients and closed supports. It is convenient to assume that all spaces are connected. Liao's theorem is an immediate consequence of the following fixed point theorem.

Theorem 2. Let $\left(S^{1}, Y\right)$ and $\left(S^{1}, X\right)$ be two transformation groups and let

$$
f:\left(S^{1}, Y\right) \rightarrow\left(S^{1}, X\right)
$$

Received by the editors December 26, 1958. 